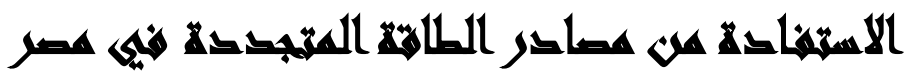 \\ اللحت هن التخغيراهت المنلخهي
}

\section{[19]}

نادر البير فانوس(')- هشام إبراهيم القصاص(") أبو بكر عبد الحميد محمد عمر(r)

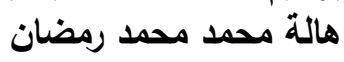

() كلية التجارة، جامعة عين شمس r ب) معهد الدراسات والبحوث البيئية، جامعة عين شمس رمس

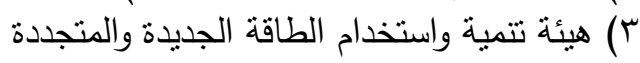

\section{المستخلم:}

تهدف الدراسة إلي الاستفادة بما نتتنع به مصر من ثراء واضح فى مصادر الإد الطاقة

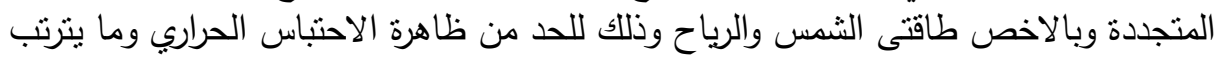

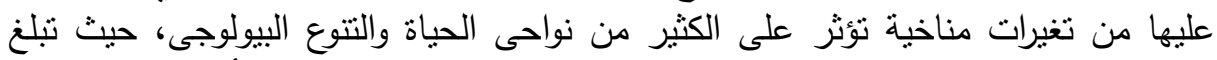

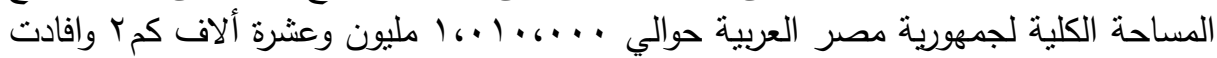

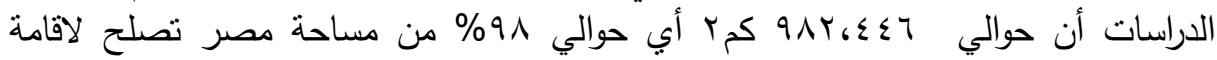

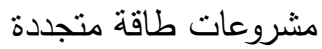

تتمثل أهمية الدراسة انها نتتاول موضعاً يرتبط بتأمين إمدادات مصر من الطاقة وكذللك

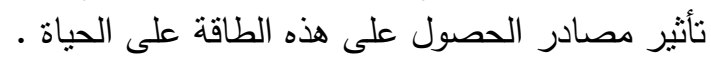

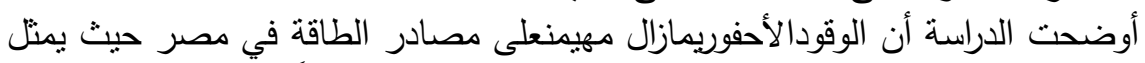

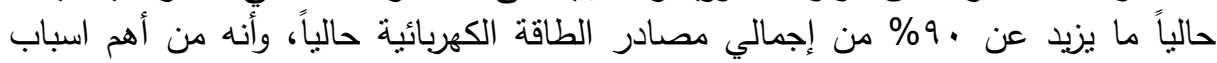
الاحترار العالمي والتغيرات المناخية.

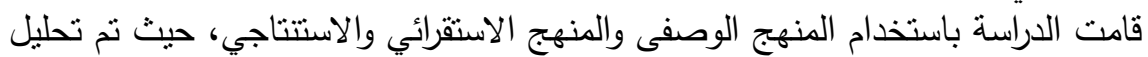

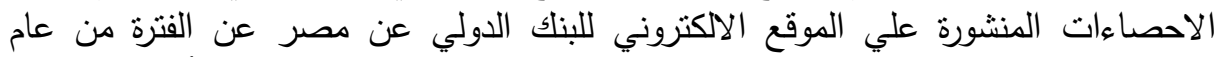

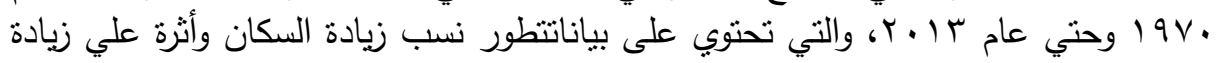

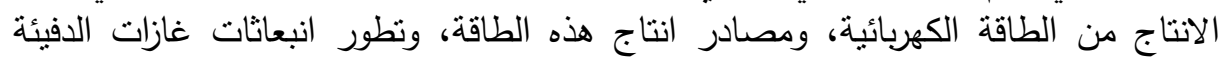

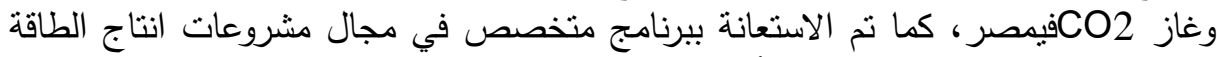

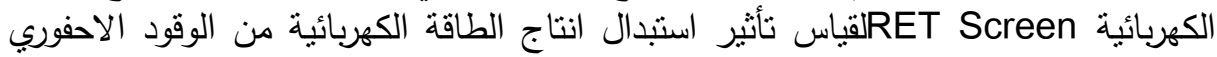


ومن أهم نتائج تحليل البيانات الخاصة بمصر عن الفترة من عام • 19V وحتي عام

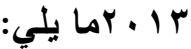

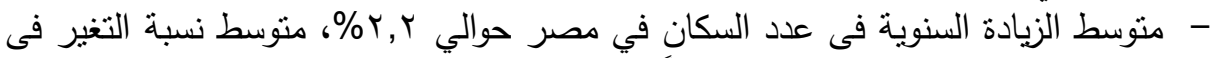

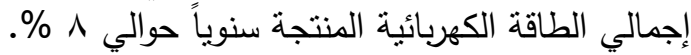

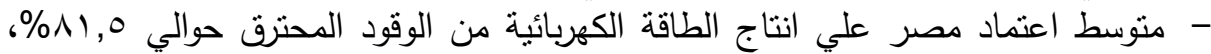

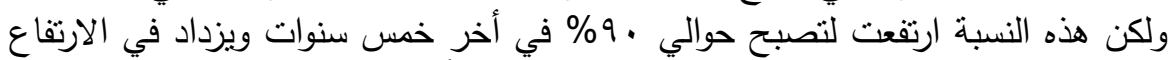
بسبب التأخر في تتفيذ استراتيجية الطاقة المتجددة وأيضاً انخفاض النتاج النات المحطات المائية.

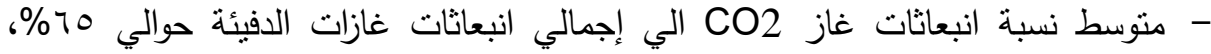
متوسط نسبة انبعاثات غاز CO2 من محطات انتات التات الكهرباء الي إجمالي انبعاثات

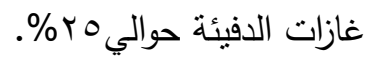
- استبدال مشروع طاقة أحفورية بمشروع طاقة متجددة بقدرة (1) ميجاوات سوف يعمل في

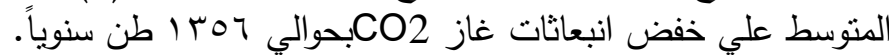

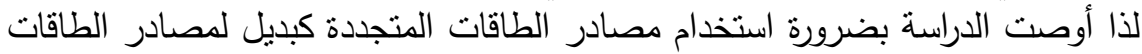
الاحفورية للحد من مخاطر التغيرات المناخية.

\section{Xaxis}

تأمين إمدادات الطاقة وبالاخص الطاقة الرخيصة هي شروط رئيسية لنمو الاقتصاد العالمي، وتقدر الوكالة الدولية للطاقة أن أسعار النفط ستستمر فى الزيادة ومالم تتخذ تدابير

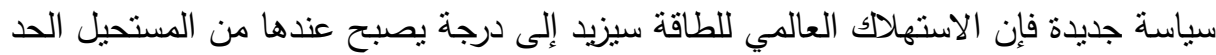
من ظاهرة الاحتباس الحراري، وينبغي إدماج إنتاج الطاقة إلى مصادر الطاقة المتجددة والتخلي عن استخدام النفط فى أقرب وقت ممكن(Katainen, 2013).

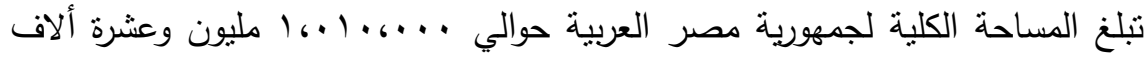

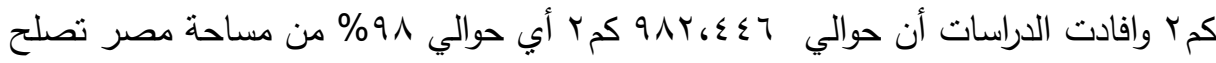

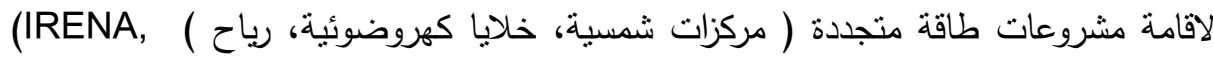

أفاد العلماء أن مستويات غازات الاحتباس الحراري تواصل الارتفاع فى الغلاف الجوي، وأن مستويات غاز ثاني أكسيد الكربون اليوم هي الأعلى منذ أكثر من ل. ..... .. سنة، 
وهناك ارتباط واضح بين مستويات ثاني أكسيد الكربون ودرجات الحرارة فى العالم، وان هذا

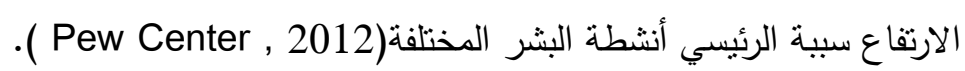

اصبح هناك اتجاة عالمي للاهتمام بالطاقات النظيفة وكفاءة استخدام الطاقةولكن لا نزال النال

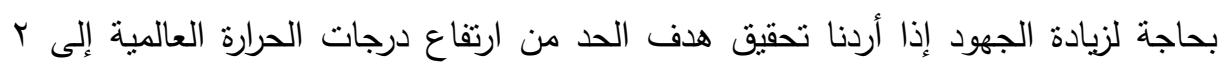

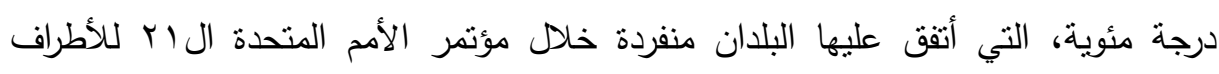

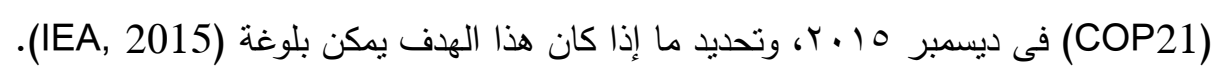

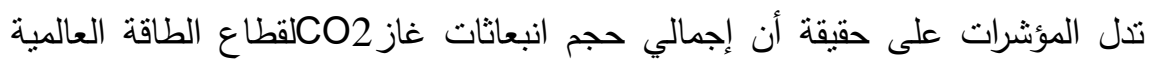

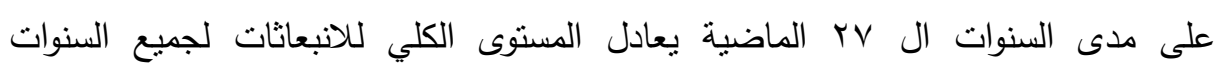
السابقة) (IAEA, 2016).

فى ظل التحديات المتزايدة الناجمة عن تغير المناخ، ونضوب موارد الوقود الأحفوري

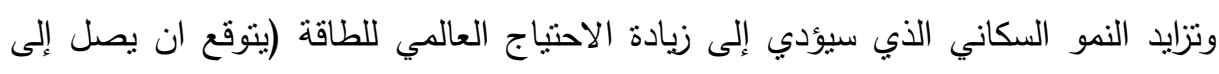

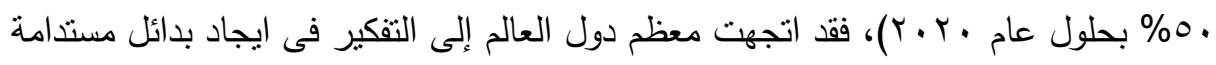

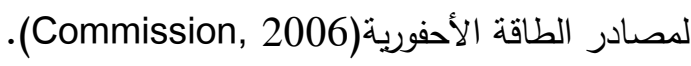
أثنار تقرير التقويم الرابع للهيئة الحكومية الدولية المعنية بتغير المناخ إلى بعض التورية التوقعات بشأن المناخ فى المستقبل حيث:

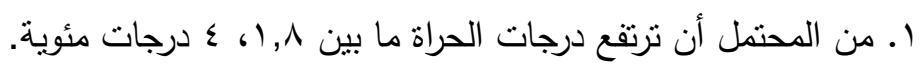

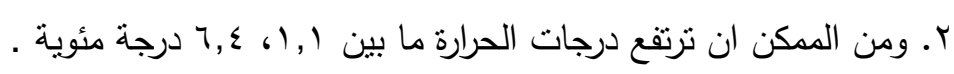

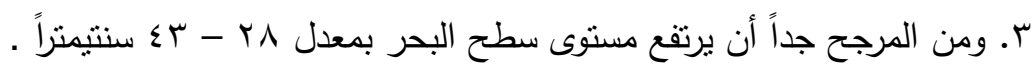

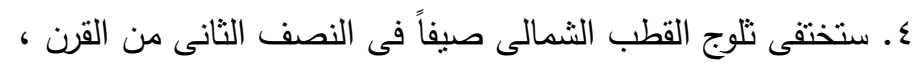
ه. من المرجح جداً زيادة الموجات الحارة . 7. من المرجح جداً زيادة حدة العواصف الادة الستوائية. 


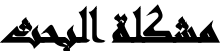

نظرأ لارتباط خطط التتمية بالطاقة وتأثير الطاقة علي التغيرات المناخية ـ فقد نم عمل

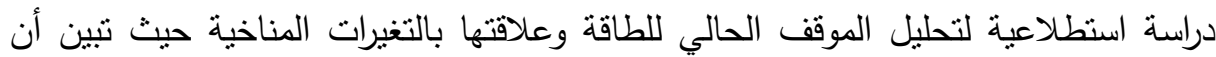
هناك تهديدات لقطاع الطاقة في مصر حيث: (1) تواجه مصر تحدي في توفير موارد كافية من مصادر الطاقة وعلى الأخص البترول

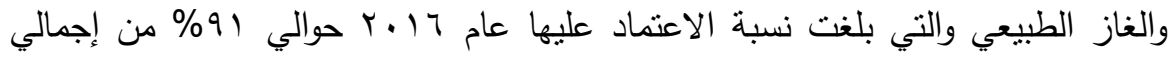

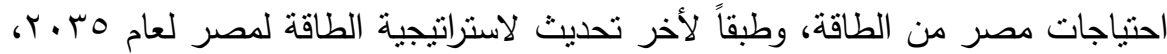
من المتوقع أن تصبح مصر مستورد دائم للبترول والغاز خلال مدة لن تجاوز عدة سنوات من بدايات العقد الثالث من هذا القرن، كما تعد مشكلة توفير الطاقة من المشاكل الهامة

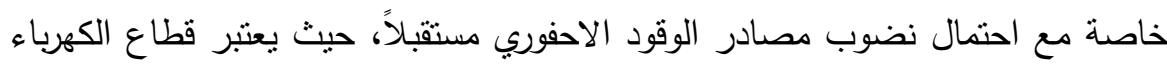

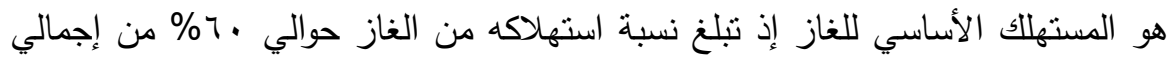

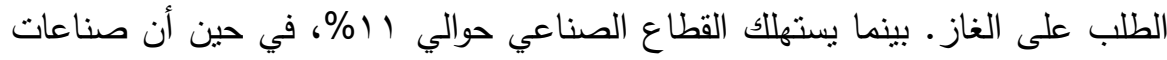

الأسمدة والأسمنت تستهلكان نسبة تبلغ · ( \% و \% على التوالي .Anon)، (2016.

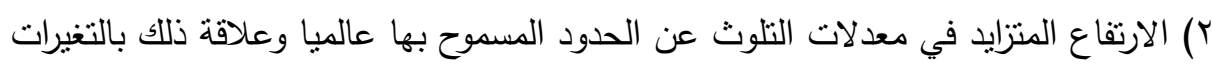
المناخية، والتي من أهم أسبابها استخدام المصادر التقليدية فيتوفير الطاقة كالبترول والغاز

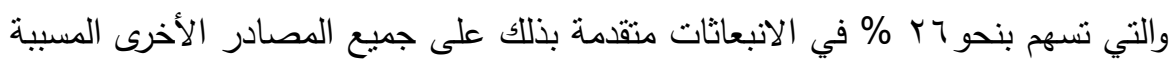
لانبعاثات الغازات الدفيئة (مصطفي منير ، (2013).

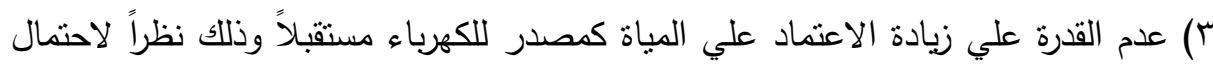
انخفاض نصيب مصر من المياة بسبب انشاء سد النهضة بأثيوبيا .

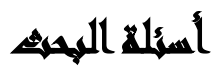

• ما مدي تأثير مصادر انتاج الطاقة الكهربائية على التغيرات التغيرات المناخية ؟

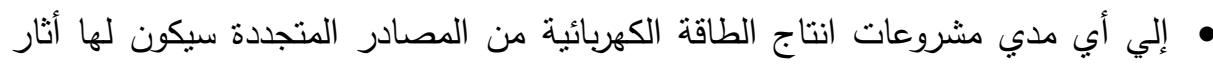
ايجابية للحد من ظاهرة الاحتباس الحراري ؟ 
• ما مدي إمكانية استخدام تكنولوجيا الطاقات المتجددة كبديل استراتيجي لمصادر الطاقة

$$
\text { الاحفورية؟ ملامي }
$$

\section{هزوبياهت المهيث}

توجد علاقة بين انتاج الطاقة الكهربائية من مصادر الطاقة المتجدة ( شمس ورياح ) والحد من التغيرات المناخية .

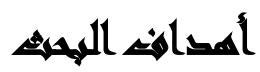

بيان مدى أهمية مشروعات انتاج الطاقة الكهربائية من المصادر المتجددة كبديل

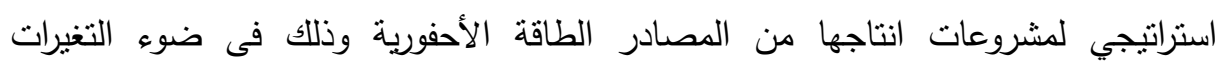
المناخية.

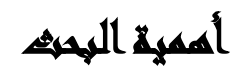

تتبع أهمية هذا البحث من الأهمية التي تحتلها ظاهرة التغيرات المناخية وأثارها المختلفة علي الكائنات الحية، وانه يجب نتجيع المشروعات التي تحقق التنمية المستدامة والحفاظ على التى

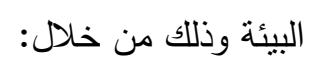
• ربط البحث العلمي بأهداف وخطط التنمية . • • تشجيع المشروعات التي تحقق التتمية المستدامة والحفاظ علي البيئة . • دعم الحد من ظاهرة التغيرات المناخية وأثارها علي صحة الانسان والبيئة وذلك من خلال استخدام مشروعات الطاقة المتجددة.

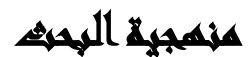

المنهج المنبع هنا هو المنهج الوصفي الذي يسعي إلى تتخيص ووصف موضوع

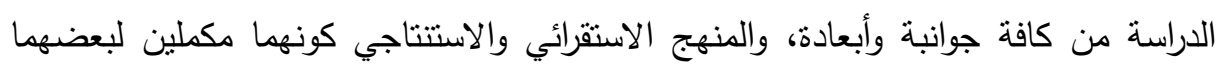

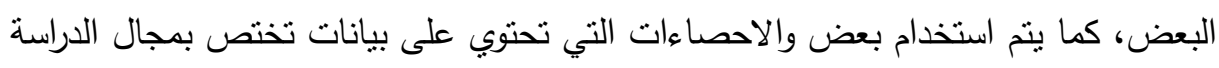

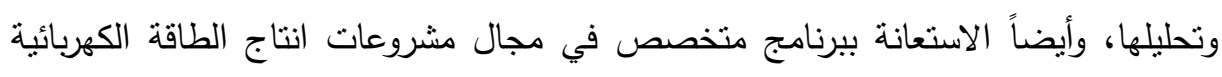

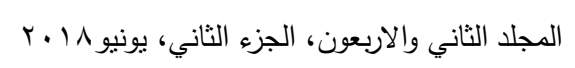


RET Screen

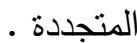

\section{هحط الهي المهمث}

• الحد الموضوعي: مشروعات انتاج الطاقة الكهربائية من مصادرها المتعددة، مع

$$
\text { التركيز علي المصادر المتجددة ( الرياح والثمس ). }
$$$$
\text { • الحد المؤسسي: قطاعالكهرباء والطاقة . }
$$

• الحد المكاني: التغيرات المناخية العالمية وأثارها على مصر •

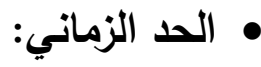

• فترة البيانات التاريخية لتعداد السكان، وإجمالي الطاقة الكهربائية المنتجة ومصادرها،

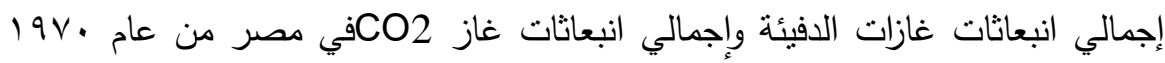

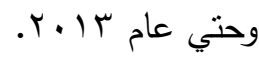

• فترة التوقعات المستقبلية لإنبعاثات غازات الدفيئة من مشروعات انتاج الكهرباء في مصر

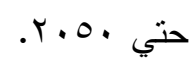

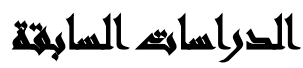

دراسة حول الطاقات المتجددة كمدخل لتحقيق التتمية المستدامة في الجزائر ) فروحات،

(2012، تهدف الدراسة الإجابة عن الإثكالية التالية : فيما يتمنل واقع ومستقبل الطاقة

المتجدة في الجزائر؟ ومامدى مساهمةهذا النوع من الطاقات فيتحقيق التتمية المستدامة؟ الإنج دراسة عن آفاق الطاقة المتجددة في مصر : فرص الخروج من شبح نضوب النداب الطاقة

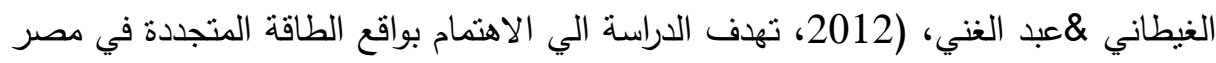

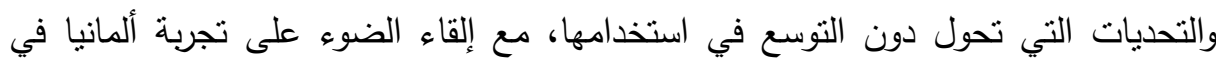
صناعة الطاقة المتجددة وكيف يمكن الاستفادةمنها. دراسة حول استراتيجية مقترحة لامكانية استخدام الطاقة الثمسية في توليد الكهرباء في مصر )عمران، (2014، تهذف الدراسة الي الاستفادة بما تتمتع به مصر من ثراء واضح في لإنه 534

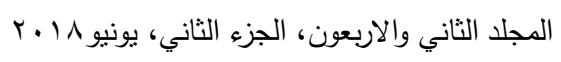


مصادر الطاقة المتجددة وذللك للتغلب علي ازمة مصر في مجال الطاقة الاحفورية من خلد

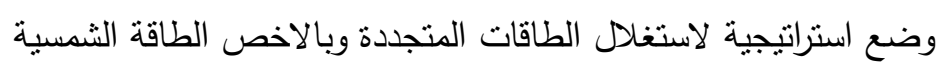
دراسة حول اقتصاديات الطاقة الثمسية كطاقة متجددة والاثار الاقتصادية لاسنتمارها في مصر (فواز، (2014 )، تهدف الدراسة الي محاولة الاجابة علي التسؤلات التالية:

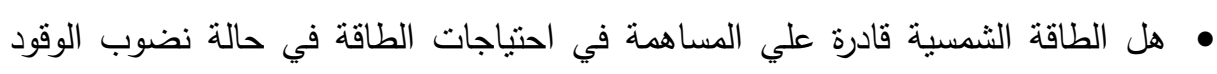
الاحفوري؟

• هل مصادر الطاقة الاحفورية الحالية كافية لاحتياجات المستقبل في العالم وفي مصر؟. • • هل الطاقة الثمسية قادرة علي نلبية احتياجات المستقبل من الطاقة؟. • هل استثمار تكنولوجيا تطبيقات الطاقة الثمسية يساعد علي احداث تتمية التصادية

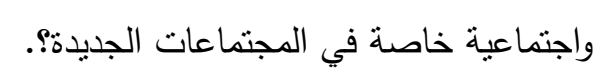

• ما هي المنافع والتكاليف المالية من استثمار تكنولوجيا الطاقة الثمسية في مصر؟؟

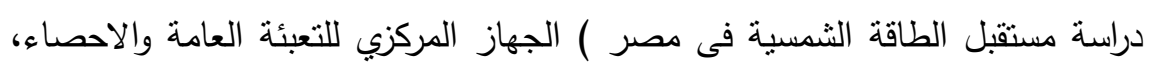
(2016، تهدف الدراسة الي التعرف على مصادر الطاقة الجديدة ومنها الطاقة الثمسية، الوقوف على الوضع الحالى لحجم وتكلفة الطاقة الثمسية فى مصر، المشروعات المستقلية الخاصة باستدامة الطاقة الثمسية وخاصة بالمجافظات المقترح التوسع فى استدامة الطاقة به،

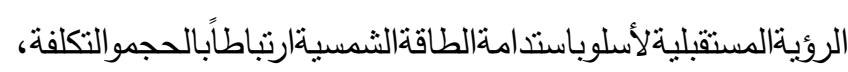
الفرصالمتاحةو المحافظانالمناسبةلتوسيعاستدامة الطاقةالثمسية. تقرير حول الطاقة وتلوث الهواء(IAEA, 2016)، تعكس هذه الدراسة رؤية وكالة الطاقة

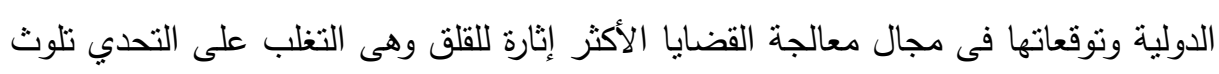
الهواء بالكامل، حيث نم إعدادها لمساعدة صناع القرار وتوفير التحليل القائم على الأدلة وتقديم المشورة بشأن سياسات الدولية تجاه التتمية والطاقة حيث تسعى وكالة الطاقة الدولية لمساعدة جميع دول العالم في التغلب على الآثار البيئية السلبية الناجمة عن استخدام الطاقة.

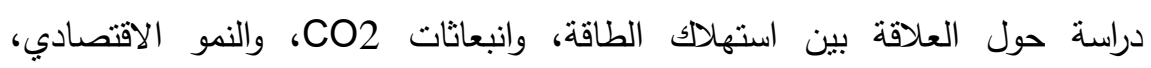
والعلاقات التجارية الخارجية في قبرص ومالطا(Taylor;, 2016)، تهدف الي دراسة العلاقة على المدى الطويل والسبيية بين انبعاثات الكربون واستهلاك الطاقة ونسبة العمالة، ونسبة

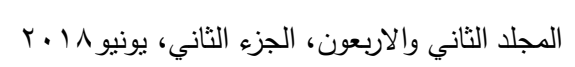


التجارة الخارجية، والنمو الاقتصادي في جزيرتي قبرص ومالطا، وذلك خلال الفترة من

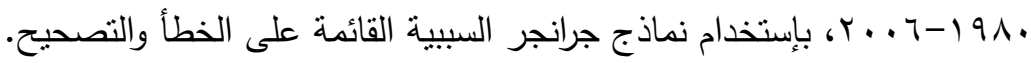

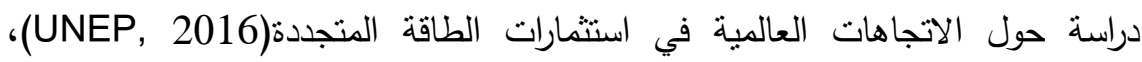

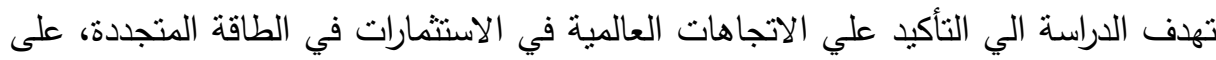

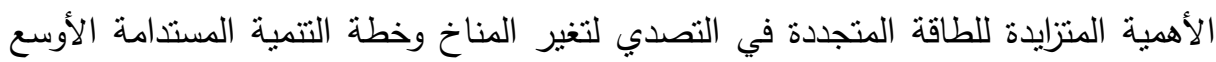
نطاقا، وتحديد الفرص الجديدة في الاستثمار في الطاقة المتجددة، لأن العالم يجب أن بستمر

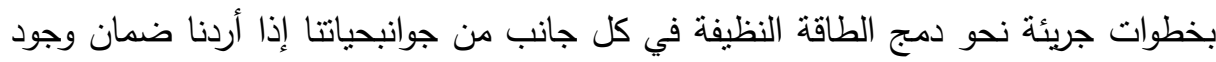

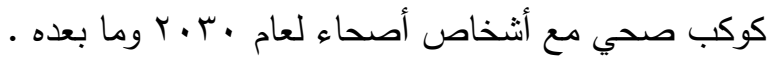

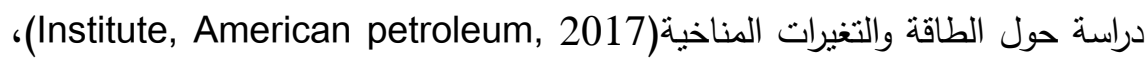
تهدف الي إيضاح الدور الفعال الذي يلعبة قطاع إنتاج وتكرير النفط والغاز الطبيعي في الولايات المتحدة الامريكية في خفض انبعاثات غازات الدفيئة ـ كانت انبعاثات ثاني أكسيد

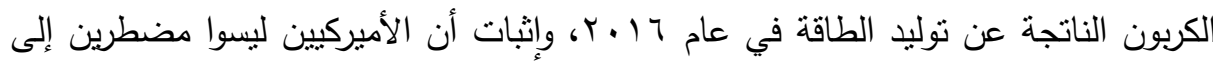
اتخاذ خبار زائف بين استخدام موارد الطاقة في بلادنا وحماية البيئة. دراسة حول مؤشرات تغير المناخ Climate action network)، (2018، تهدف إلي بوانه قياس نجاح اتفاق باريس 10 • ب من خلال تتفيذ أهداف التخفيف على المستوى الوطني، حيث

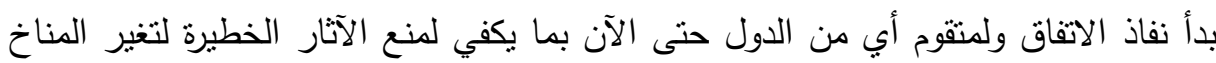

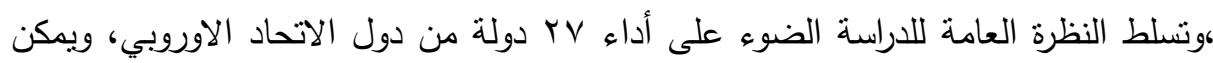
الاطلاع على نتائج جميع البلدان الrه الموقعة علي الاتفاقية. 


\section{اللخغيرايت المنالمية}

أسباب التغيرات المناخية: يوجد العديد من الاسباب التى تؤدى الي تغير المناخ منها ما هو طبيعى ومنها ما هو غير طبيعى.

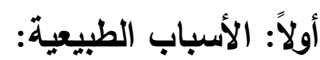

• التغيرات التي تحدث لمدار الأرض حول الثمس و ما ينتج عنها من تغير في كمية الإشعاع الثمسي الذي يصل إلى الأرض. وهذا عامل مهم جداً في التغيرات المناخية

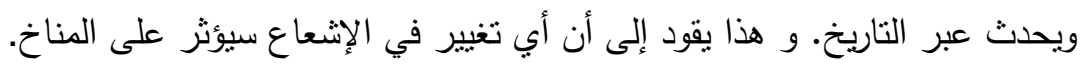

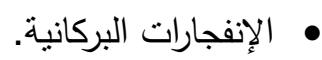

$$
\text { • التغير في مكونات الغلاف الجوي. }
$$

ثانياً: الأسباب غير الطبيعية: ناتجة من النشاطات الإنسانية المختلفة مثل : قطع الأعثاب و

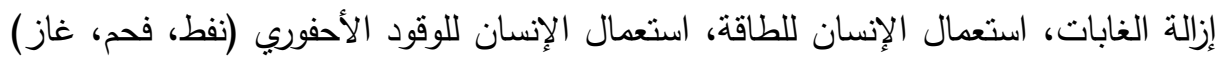

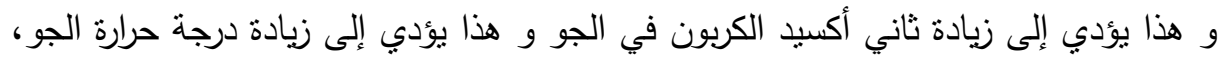

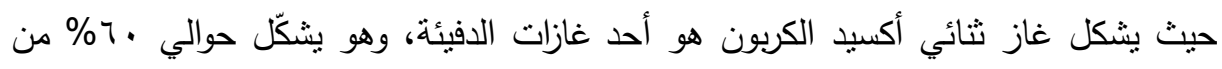
الغازات المؤثّرة على الاحتباس الحراري( Meehl, Gerald, 2004).

\section{الأثار المترتبة على التغيرات المناخية:}

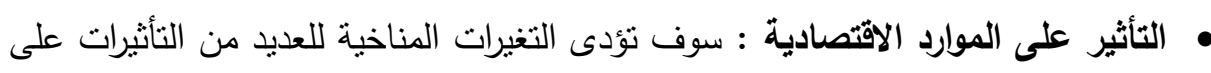
جمهورية مصر العربية ومنها التأثير على الموارد المائية حيث يزداد الضغط على على مصادئ مصادر المياه وزيادة معدل الاستهلاك خاصة في الزراعة والصناعة، يحدث تغير في كميات

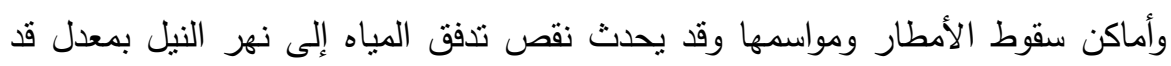

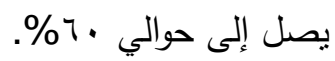

• التأثير على الزراعة والثروة الحيوانية ومصادر الغذاء حيث تتقص إنتاجية المحاصيل الزراعية ومصادر الغذاء، تتغير خريطة التوزيع الجغرافي للمحاصيل الزراعية، زيادة معدلات التصحر ،كما أن زيادة الحرارة تزيد من معدلات نآكل التربة.

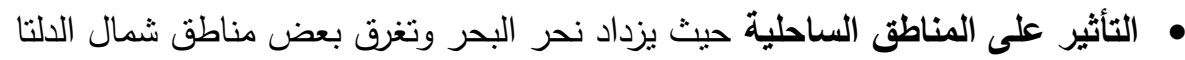

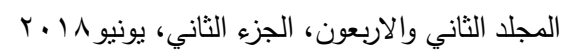


• التأثير على السياحة حيث تتدهور حالة بعض الاماكن الاثرية، تتأثز الثعب المرجانية.

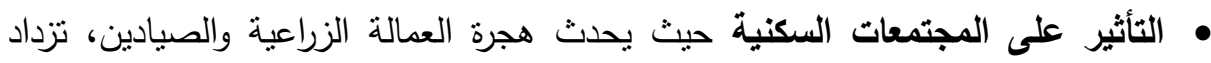
البطالة، زيادة معدلات الأمراض و ارتفاع معدلات الوفيات لدى الأطفال .

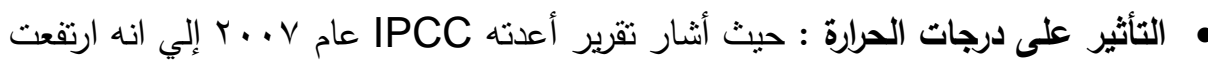
درجة حرارة اليابسة منذ عام 9 1979 بضعف مقدار ازدياد درجة حرارة المحيطات

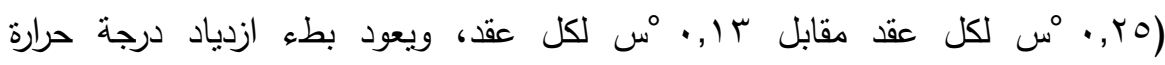

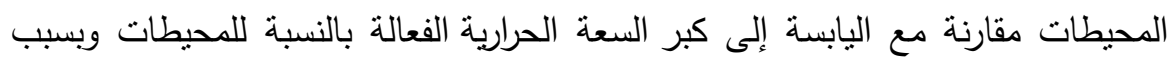

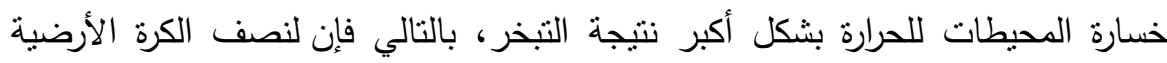

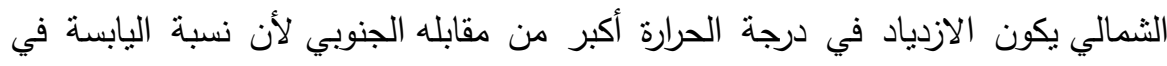

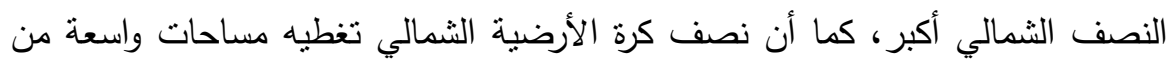

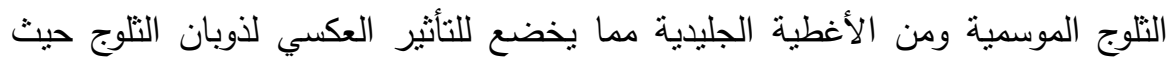
ينخفض معامل الارتداد الإشعاعي في تلك المناطق مما يعني امتصاص أكبر للحرارة. انبعاثات الكريون العالمية وتكلقتها الاقتصادية: بلغت انبعاثات الأنشطة الاقتصادية في العالم حسب تقرير مؤسسة Watch World الأمريكية، نحو Y,A مليار طن من غاز

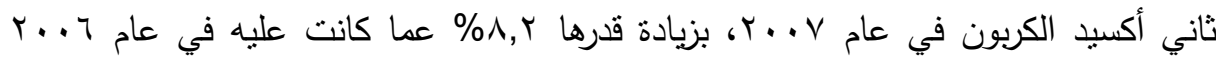

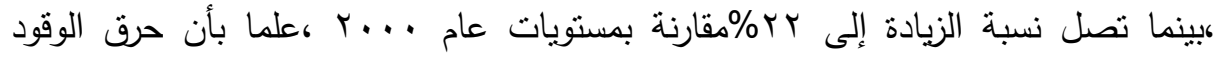

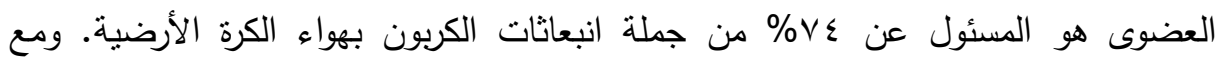

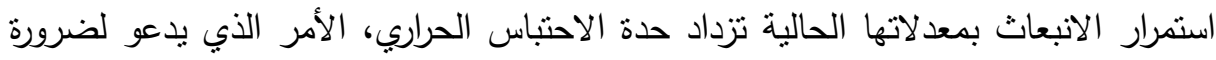

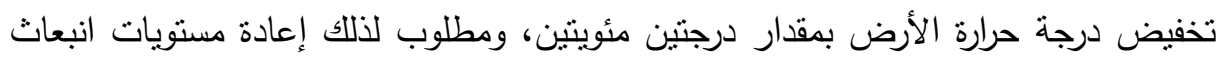

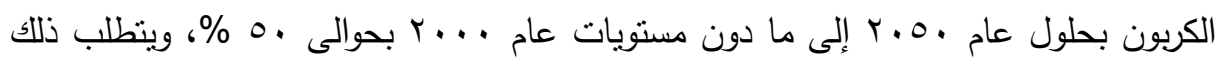

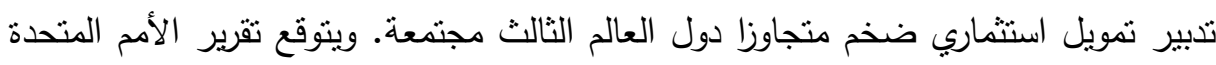

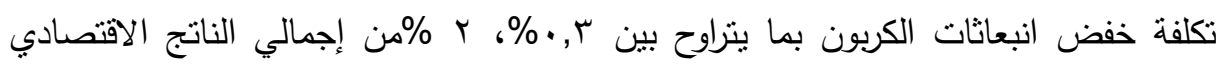

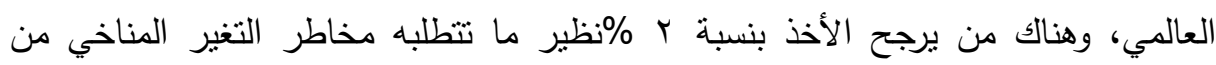


الاستثمارات لتخفيض نسبة الكربون المطلوبة. أما في حالة التراخي عن اتخاذ الإجراءات

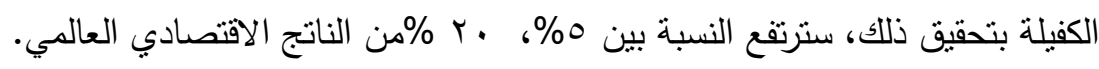
مصادر انتاج الطاقة الكهربائية: إن علية نوليد أو إنتاج الطاقة الكهبائية هي فى بـ الحقيقة عملية تحويل الطاقة من شكل إلى آخر حسب مصادر الطاقة المنوفرة فى مراكز

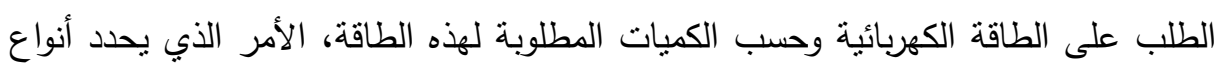

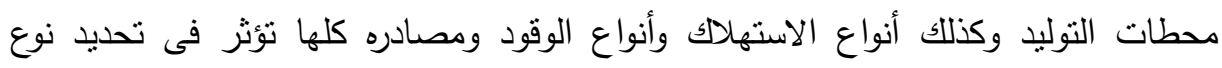

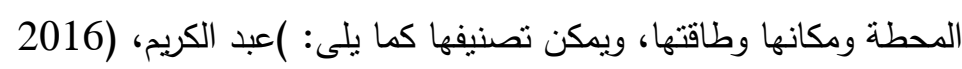

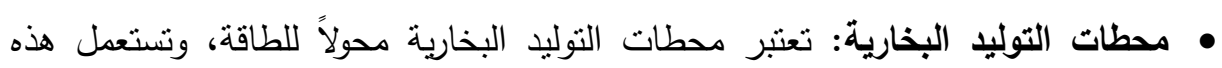

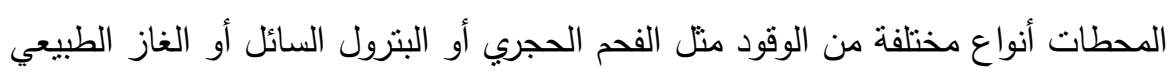

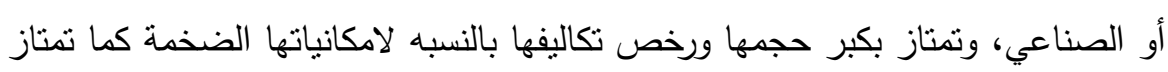
بامكانية استعمالها فى تحليه المباة المالحة. • محطات التوليد النووية: تعد محطات التوليد النووية نوعا من محطات التوليد الحرارية

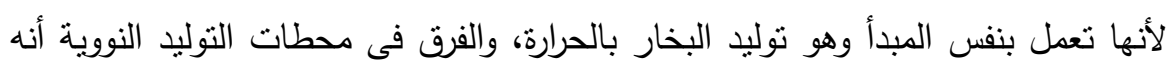
بدل الفرن الذي يحترق فيه الوقود يوجد هنا مفاعل ذري تتولد فى الحرارة نتيجة انشطار

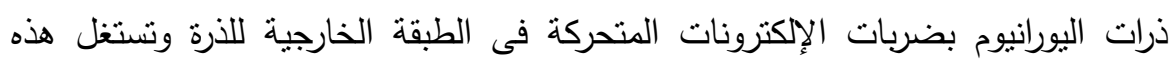

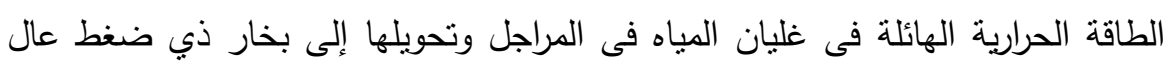

$$
\text { ودرجة مرتفعة جدا . }
$$

• محطات التوليد المائية: يمكن التفكير بانتاج الطاقة من المصادر المائية حيث نوجد المياه

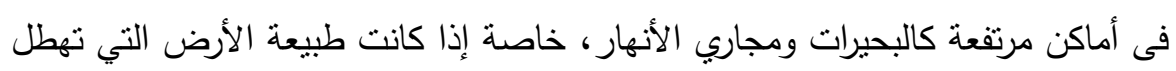

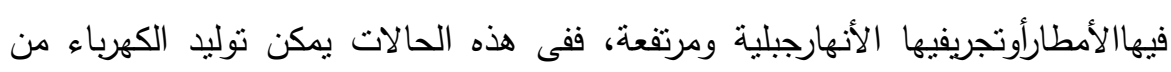

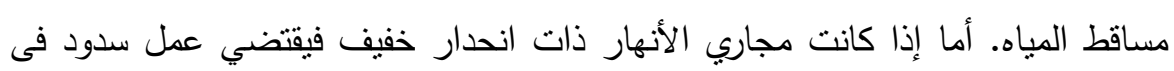
الأماكن المناسبة من مجرى النهر لتخزين المياه. • محطات التوليد من المد والجزر: المد والجزر من الظواهر الطبيعية المعروفة عند سكان

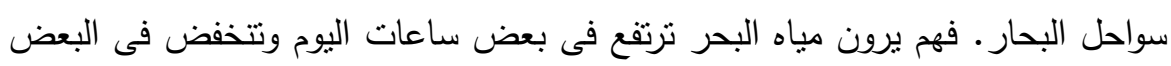

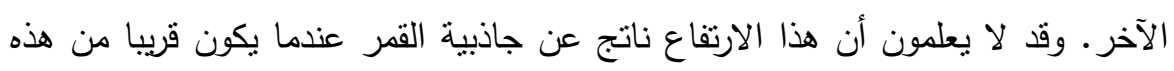

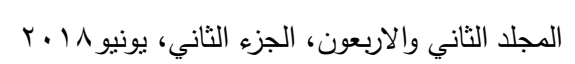


السواحل وان ذلك الانخفاض يحدث عندما يكون القمر بعيدا عن هذه السواحل، أي عندما يغيب القمر • محطات التوليد ذات الاحترلق الداخلي (ديزل - غازية): محطات التوليد ذات الاحتراق

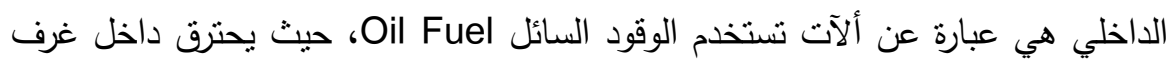

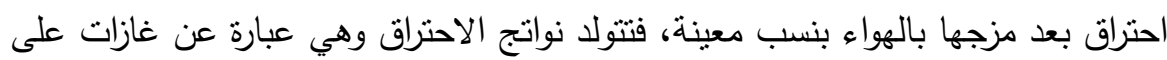

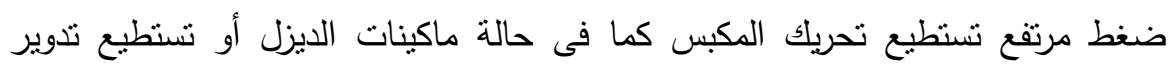
التوربينات حركة دورا نية كما فى حالة التوربينات الغازية.

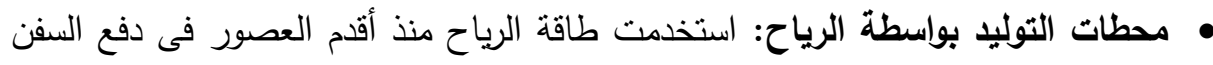

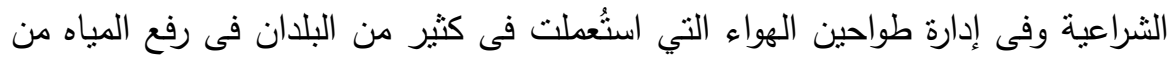

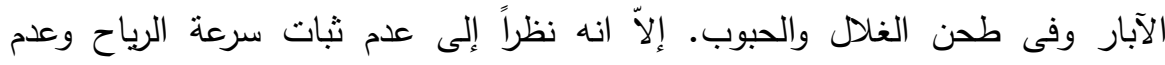

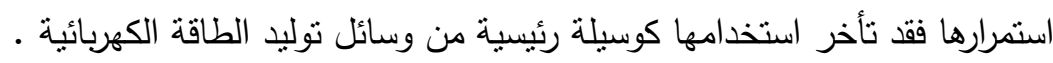

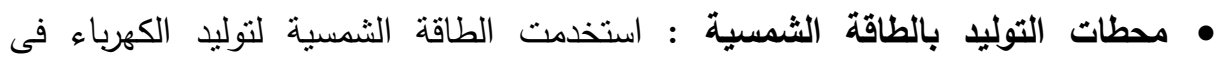
تطبيقات عديدة منها محطات توليد الكهرباء وتحلية المياه، وتتغيل إثارات المرور وإنارة الثوارع، وتتغيل بعض الأجهزة الكهربائية مثل الساعات، والآلات الحاسبة، وتتشغيل ونيل

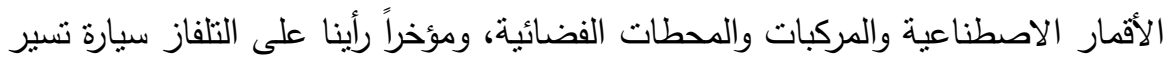
بالطاقة الثمسية. مزيج الطاقة الكهربائية المنتجة في مصر: يوضح نقرير الثركة القابضة لكهرباء مصر مصادر انتاج الطاقة الكهربائية في مصر خلال عام 17 ـr، حيث بتضح اعنماد مصر علي انتاج الطاقة الكهربائية من الوقود المحترق، وهذا الوقود بأنواعة المختلفة سبب

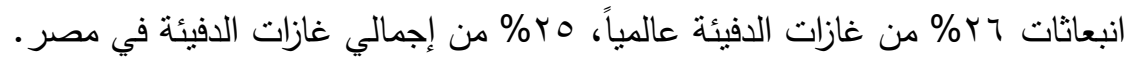


نادر ألبير فانوس وآخرون

جدول( (1): مزيج انتاج الطاقة الكهربائية في مصر عام 17 ـ الط

\begin{tabular}{|c|c|c|c|c|}
\hline الطن المصادة المنتجة & الطن المحطة المنتجة & من الوقود المحترقة & إجمالى الطاقة & البيان \\
\hline TGKYT & $1 \pi, 0 \leqslant 0$ & $1 V .600$. & 1NT,rT. & (جيجاواتة ساعة الطنتجة ) \\
\hline$\% 1, r$ & $\% \vee, r$ & $\% 91,0$ & $\% 1 \ldots$ & النسبة المئوية \\
\hline
\end{tabular}

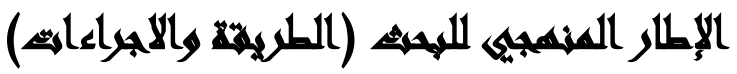

وصف أداة البحث: تم استخدم تحليل النطور التاريخي للبيانات المنشورة بالموقع الالكتروني للبنك الدولي ( data.worldbank.org) عن مصر والتي تخص نطور انتاج الطاقة الكهربائية ومصادر انتاجها كنتيجة لزيادة عدد السكان، وبيان تأثنير الزيادة في انتاج الطاقة الكهربائية وبالاخص من الوقود الاحفوري علي زيادة انبعاثات غازات الدفيئة وبخاصة غاز ثاني أكسيد الكربون (CO2)، وتم نوذللك وفق الاطار الزماني والمكاني التالي:

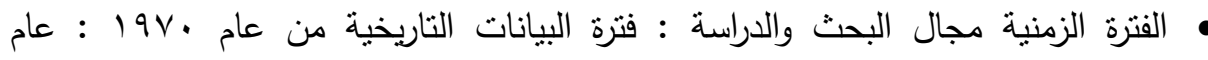

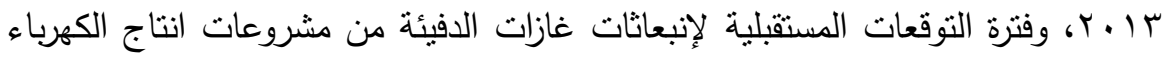

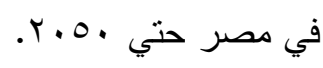
• الاطار الجغرافي للبحث والدراسة : جمهورية مصر العربية. كما تم أيضاً استخدام برنامج متخصص في مجال مشروعات الطاقة (RET Screen) لحساب الوفر في انبعاثات غاز CO2 عند استبدال استخدام الطاقة المعتمدة على المواد الأحفوري والتي تسبب في معدلات تلوث كبير والانتقال الي استخدام طاقة متجددة كطاقة الثمس وطاقة الرياح. 


\section{إجراءاهي الهمشه}

أداة البحث: تم تصميم جداول تحتوي علي البيانات المنشورة علي موقع البنك الدولي عن مصر بشأن تطور الزيادة اعداد السكان وتأثيرة علي انتاج الطاقة الكهربائية وعلاقتة بزيادة

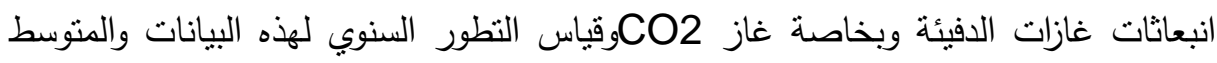
العام كما بلي : حاني

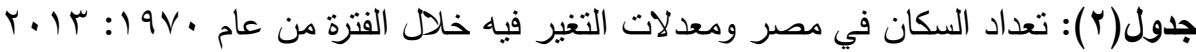

\begin{tabular}{|c|c|c|}
\hline معدل التغير (\%) & إجمالي عدد السكان ( مليون نسمة ) & 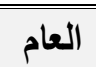 \\
\hline ב- & $r_{0}$ & $19 V$. \\
\hline$\% r$ & $r$ & $19 \times 1$ \\
\hline$\% r$ & $r v$ & $19 V Y$ \\
\hline$\% r$ & TV & $19 V Y$ \\
\hline$\% r$ & rᄉ & $19 \vee \varepsilon$ \\
\hline$\% r$ & rq & $19 \times 0$ \\
\hline$\% r$ & $\varepsilon$ & $19 \times 7$ \\
\hline$\% r$ & $\xi 1$ & $19 V V$ \\
\hline$\% r$ & $\varepsilon Y$ & $19 \vee \wedge$ \\
\hline$\% r$ & $\varepsilon r$ & $19 \vee 9$ \\
\hline$\% r$ & $\varepsilon \varepsilon$ & 191. \\
\hline$\% r$ & $\leqslant 0$ & 1911 \\
\hline$\% r$ & $\varepsilon 7$ & $191 Y$ \\
\hline$\% r$ & $\varepsilon \wedge$ & $19 \wedge r$ \\
\hline$\% r$ & $\sum 9$ & $191 \varepsilon$ \\
\hline$\% r$ & 0. & 1910 \\
\hline$\% r$ & OY & 1917 \\
\hline$\% r$ & Or & $19 \wedge V$ \\
\hline$\% r$ & 00 & 1911 \\
\hline
\end{tabular}


نادر ألبير فانوس وآخرون

تابع جدول(r):

\begin{tabular}{|c|c|c|}
\hline معدل التغير (\%) & إجمالى علد السكان ( مليون نسمة ) & العام \\
\hline$\% r$ & 07 & 1919 \\
\hline$\% r$ & OV & 199. \\
\hline$\% r$ & 09 & 1991 \\
\hline$\% r$ & 7. & 1994 \\
\hline$\% Y$ & 71 & 1994 \\
\hline$\% Y$ & $7 r$ & 1998 \\
\hline$\% Y$ & Tะ & 1990 \\
\hline$\% Y$ & 70 & 1997 \\
\hline$\% Y$ & 77 & $199 \mathrm{~V}$ \\
\hline$\% Y$ & $7 V$ & 1991 \\
\hline$\% Y$ & 79 & 1999 \\
\hline$\%$ \% & $V \cdot$ & r... \\
\hline$\%$ \% & V) & $r \ldots 1$ \\
\hline$\% r$ & $\overline{v T}$ & $r \ldots r$ \\
\hline$\%$ \% & $V \varepsilon$ & $r \ldots r$ \\
\hline$\% Y$ & Vo & Y... \\
\hline$\% Y$ & $\overline{V V}$ & $r \ldots o$ \\
\hline$\% Y$ & $\overline{\nabla \wedge}$ & $r \ldots T$ \\
\hline$\% r$ & A. & $r \ldots V$ \\
\hline$\% r$ & $\lambda 1$ & $r \ldots \lambda$ \\
\hline$\% r$ & $\Lambda Y$ & $r \ldots q$ \\
\hline$\% r$ & $\Lambda \varepsilon$ & $r \cdot 1$. \\
\hline$\% r$ & $\Lambda \mathrm{T}$ & $r \cdot 11$ \\
\hline$\% r$ & $1 \mathrm{\Lambda \Lambda}$ & $T \cdot I T$ \\
\hline$\% r$ & 9. & 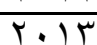 \\
\hline \%Y, & , & \\
\hline
\end{tabular}

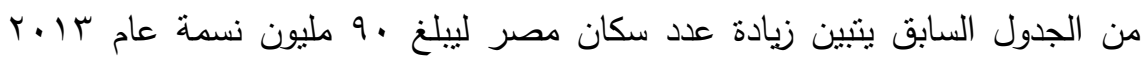

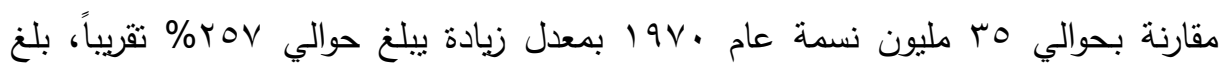

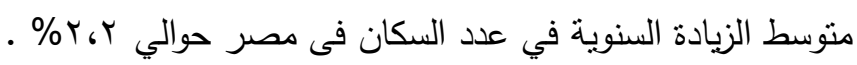




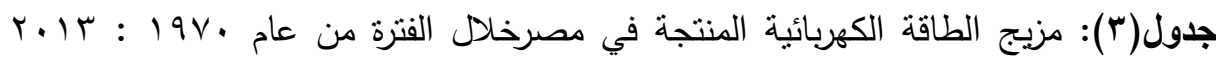

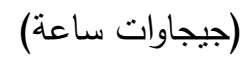

\begin{tabular}{|c|c|c|c|c|c|}
\hline 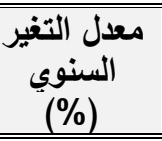 & الطأقة الكهريائية & التناج الكهرياء & 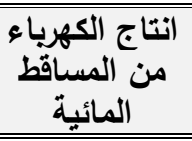 & انتاج الكهرياء & العام \\
\hline- & 7.910 & . & $\varepsilon .79$ & T.YTO & $19 V$. \\
\hline$\%\urcorner$ & VGTYT & . & $0, \cdot \leqslant 1$ & T.YAT & 1971 \\
\hline$\% 1$ & $V_{6} r \wedge \varepsilon$ & . & 0,109 & TGYTO & $19 V Y$ \\
\hline$\% 1$ & $V_{6} \varepsilon T_{0}$ & . & 0,107 & r. TV9 & $19 \mathrm{Vr}$ \\
\hline$\% 10$ & 1,019 & . & T.ITY & r.rqV & $19 V \varepsilon$ \\
\hline$\% 10$ & $9, \wedge \ldots$ & · & 7.89. & $r_{6 .}{ }^{\prime}$ & 1980 \\
\hline$\% 19$ & $11,7 \leq 7$ & . & $\Lambda_{6} \ldots r$ & $r, T \leqslant r$ & $19 V 7$ \\
\hline$\% 17$ & $1+1.01 \mathrm{~V}$ & . & $96 .+1$ & $\varepsilon_{6} \varepsilon \vee 9$ & $19 \mathrm{VV}$ \\
\hline$\% \backslash 1$ & $10, .14$ & . & 9.940 & $0, . V \lambda$ & $19 \vee \wedge$ \\
\hline$\% 9$ & 17.509 & . & 967.1 & 7.801 & $19 \vee 9$ \\
\hline \%) & $1 \Lambda_{6} \leqslant Y q$ & . & $9,1 \ldots$ & 1.7Y & 191. \\
\hline$\%$ & $r \cdot \sigma V \leqslant \Lambda$ & . & $1.6 r 17$ & 1.6045 & 1911 \\
\hline$\%$ & rTGOT & · & $1 \cdot 6 \varepsilon \wedge \varepsilon$ & $1 T_{6} 1179$ & 1914 \\
\hline$\% 11$ & Yo، AVq & . & 96117 & 176.75 & $191 r$ \\
\hline$\% 1 r$ & $r q_{6} . \leqslant 9$ & . & 9.7Tr & 196817 & $191 \varepsilon$ \\
\hline$\% \wedge$ & r1, 501 & . & 1.74 & rY.V9O & 1910 \\
\hline$\% 4$ & Mr. & . & $96 Y \wedge 1$ & $r \leq 61 \wedge r$ & 1917 \\
\hline$\% 1$. & $r 4.190$ & . & 1.701 & YA,KTV & $191 \mathrm{~V}$ \\
\hline$\% r$ & $r V_{6} \wedge \leqslant 0$ & $\cdot$ & $\Lambda_{6} Y \circ 9$ & $r 9.017$ & 1911 \\
\hline$\% 0$ & $r 9,01$. & · & Q.rTr & $r \cdot r$ ron & 1919 \\
\hline$\% 0$ & $\leqslant 1,7 \leqslant 9$ & $\cdot$ & $9.9 \vee 0$ & MI.TVE & 199. \\
\hline$\% \leq$ & $\varepsilon r_{6} \varepsilon \vee \wedge$ & $\cdot$ & 9.VTr & $r r . V \leq T$ & 1991 \\
\hline$\% 0$ & $\varepsilon 0, \varepsilon \wedge r$ & . & $1 \cdot G r \cdot \varepsilon$ & TOGYYA & 1995 \\
\hline$\% \varepsilon$ & $\sum V_{6} .97$ & . & $1 \cdot 6 \leqslant 10$ & $r 4.711$ & 1994 \\
\hline
\end{tabular}


نادر ألبير فانوس وآخرون

تابع جدول(ץ):

\begin{tabular}{|c|c|c|c|c|c|}
\hline التغيرل & الجمالي النتاجة & الكهرباء المصناد & المساج الكهرياء من المائة & انتاج الكترياء & العام \\
\hline$\% r$ & $\sum \Lambda_{6} 7 . \leqslant$ & & $1.69 \times 1$ & TV.TTr & $199 \varepsilon$ \\
\hline$\% 7$ & 01, KYA & & $11 ، \Omega 1 \pi$ & $r 9.910$ & 1990 \\
\hline$\% 7$ & $0 \leqslant$ \& $\leqslant 79$ & & 11,000 & $\varepsilon r_{6} q 1 \leq$ & 1997 \\
\hline$\% 7$ & $0 V 6707$ & & 11,917 & $\sum 0,7 \mathrm{~V}$. & $199 V$ \\
\hline$\% \Lambda$ & TYGrTt & & $\mid T_{G}, T_{1}$ & 0.6110 & 1991 \\
\hline$\% 9$ & $7 V .911$ & & $10, \mathrm{rAV}$ & $0 r_{6} 79 \leq$ & 1999 \\
\hline$\% \wedge$ & 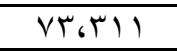 & $\cdot ., Y \varepsilon$ & $1 \leqslant 6709$ & $0 \Lambda .7 Y \Lambda$ & r... \\
\hline$\% 7$ & $V_{V} V_{6} \wedge \varepsilon \cdot$ & $.61 \mathrm{rV}$ & $14679 \mathrm{~V}$ & $7 \varepsilon_{6} \ldots 7$ & $r \ldots 1$ \\
\hline$\% \varepsilon$ & NI.YTV & . $6 \mathrm{Y} . \xi$ & $1 K_{6} \wedge 09$ & $T \Lambda_{6}, Y \cdot \Sigma$ & $r \ldots r$ \\
\hline$\% \backslash V$ & $9 \varepsilon ، 91 \%$ & . & 1146.19 & $11.0 Y 4$ & $r \ldots r$ \\
\hline$\% 4$ & $1 \ldots .9997$ & $.0 \% T$ & TrGT $\varepsilon \varepsilon$ & $\Delta V_{6} \Delta Y_{q} q$ & $r \ldots \varepsilon$ \\
\hline$\% \wedge$ & 1.1079. & $.000 Y$ & Tr.T $\varepsilon \varepsilon$ & $90, \leqslant 9 \leqslant$ & $r \ldots o$ \\
\hline$\% 7$ & $110, \varepsilon \cdot V$ & .6717 & IY.9YO & 1.16177 & $r \ldots r$ \\
\hline$\% \wedge$ & $1 \times 0,1 \times 9$ & . . & $10,01$. & $1 . \Lambda_{6} \vee \wedge \Lambda$ & $r \ldots v$ \\
\hline$\% 0$ & $1 \pi 16$. & .6941 & IE،TAY & $110 . \Omega \mathrm{TV}$ & $r \ldots \Lambda$ \\
\hline$\% 7$ & $1+96 \ldots$ & $1,1 Y \mu$ & Ir,ATr & $1 Y 06.01 \leq$ & $r \ldots q$ \\
\hline$\% \varepsilon$ & $1 \leq 0, Y Y \leq$ & $1,\{71$ & $1 \%, . r \mu$ & $1 T \cdot 6 V T$. & $r \cdot 1$. \\
\hline$\% 1$ & $1 \leq 76997$ & $1, V \cdot \varepsilon$ & $1 \% 6 . \leq 4$ & $1 Y_{6} . \leq 7$ & $r .11$ \\
\hline$\% \mathrm{~V}$ & $10 V_{6} \varepsilon+7$ & $r_{6} \ldots \varepsilon$ & 1 1 $694 \leq$ & $1 \leq Y_{6} \leqslant 71$ & $r .1 Y$ \\
\hline$\% 0$ & 17 IITYA & $1.29 V$ & $\mid r, 1 Y 1$ & 10.6 .1$. & T.MT \\
\hline & Y.ONTGKTV & $11,9 \vee 0$ & $\leq 70,1) 9$ & $r_{6} 1 \cdot 0, Y Y Y$ & الإجمالي \\
\hline & $\% 1 \ldots$ & $\% \cdot . \leqslant 7$ & $\% \backslash \wedge$ & $\% \wedge 1.0 \leq$ & النسبة \\
\hline$\% \wedge$ & \multicolumn{5}{|c|}{ متوسط معدل التغير السنوي فى إجمالى الطاقة الكهربائية المنتجة } \\
\hline
\end{tabular}

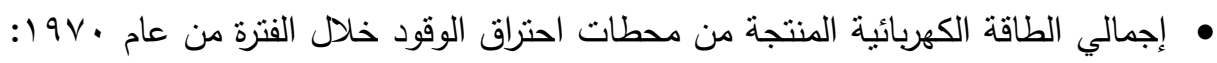

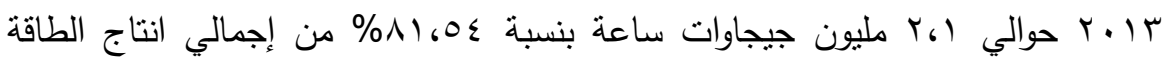
الكهربائية من مختلفة مصادر الطاقة. 
• إجمالي الطاقة الكهبائية المنتجة من محطات المساقط المائية خلال الفترة من عام

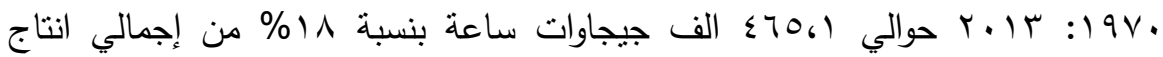
الطاقة الكهربائية من مختلفة مصادر الطاقة. • إجمالي الطاقة الكهربائية المنتجة من محطات الطاقة المتجددة خلال الفترة من عام

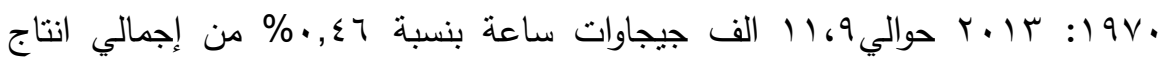
الطاقة الكهربائية من مختلفة مصادر الطاقة.

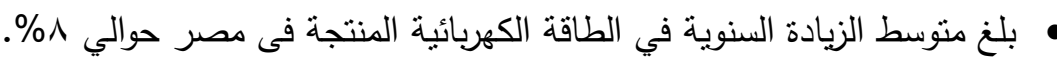

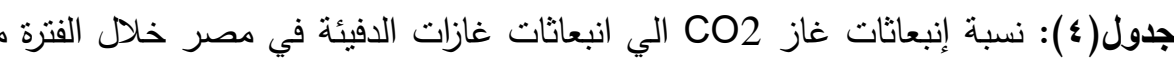

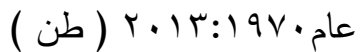

\begin{tabular}{|c|c|c|c|}
\hline من مشروعاث الكهرباء & إجمالي إنبعاثات غاز CO2 & إجمالي غازات الافيئة & العام \\
\hline r،乞० & YI.7AV & 07,101 & $19 V$. \\
\hline$r_{6} 19 V$ & $r r_{6} \cdot \Sigma V$ & $07.7 T Y$ & 1981 \\
\hline$\varepsilon_{6} \ldots \lambda$ & Y0.011 & $0961 \leqslant Y$ & $19 V Y$ \\
\hline$r_{6} \leqslant 10$ & rr6V90 & 07.7TY & $19 V \pi$ \\
\hline $2, Y 19$ & YY, Y YE & 71619. & $19 V \varepsilon$ \\
\hline $0, .9 V$ & M161... & $7 \leqslant$ ภ $\leqslant 7$ & 1980 \\
\hline 7.019 & $r \leqslant 6 \leqslant 90$ & $V .6991$ & $19 \vee 7$ \\
\hline$V_{6} 9 \leq \leq$ & rV،八7o & VY.007 & $19 V V$ \\
\hline$\Lambda_{671}$. & rq, & VT,VVY & 1981 \\
\hline $96 \vee \leqslant 9$ & $\leqslant r_{6} 9 \leqslant \Lambda$ & $V 7.101$ & 1979 \\
\hline $1.67 V V$ & $\varepsilon 0, Y \leqslant$. & $\Lambda \varepsilon_{6} \Lambda \cdot \Lambda$ & 191. \\
\hline $1 Y_{6} \leq 9$. & $0.69 \vee 9$ & $9 r_{6} \leqslant 9 V$ & 1911 \\
\hline 106.10 & $07.0 Y T$ & 996917 & 1914 \\
\hline 10617 & $0 V_{6} .94$ & 1.06949 & $191 \mu$ \\
\hline $1 \wedge 6 \% \wedge T$ & 7r.人 & $11168 V 1$ & 1915 \\
\hline$r \cdot . r \cdot \varepsilon$ & 7r.9rर & 110.019 & 1910 \\
\hline$r \varepsilon, 0 r r$ & $V \leqslant 6070$ & (T., & 1917 \\
\hline$r \varepsilon 69 \vee 9$ & $V \varepsilon_{6} \wedge 1$. & $1 Y 169 \leqslant Y$ & $191 \mathrm{~V}$ \\
\hline$r \varepsilon_{6} \cdot r_{Y}$ & $V \varepsilon, 0 Y Y$ & IYq,rYT & $19 \wedge 1$ \\
\hline$r r_{6} 0.9$ & $V Y_{6} \backslash \wedge 1$ & 1106011 & 1919 \\
\hline 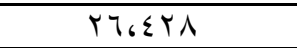 & V0.9 $\varepsilon \leq$ & $1 r q 61 \cdot r$ & 199. \\
\hline
\end{tabular}


نادر ألبير فانوس وآخرون

تابع جدول(؛) ):

\begin{tabular}{|c|c|c|c|}
\hline من مثروعاثات غاز الكهرباء & إجمالي إنبعاثات غاز CO2 & إجمالي غازات الدفيئة & 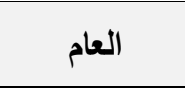 \\
\hline rV610V & $\vee \wedge 6 \vee 117$ & $1 \leq 1, \wedge \leq V$ & 1991 \\
\hline YVG YYE & A1, YY & $1 \leqslant 16779$ & 1994 \\
\hline 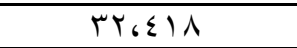 & 916107 & $10.69 \mathrm{~V}$. & 1994 \\
\hline$r \Lambda_{6} 0 \Lambda$. & NO & $1 \leqslant 76704$ & 1998 \\
\hline $1,611$. & 906 VYT & 1006040 & 1990 \\
\hline Mr.\&7T & $1 \cdot Y_{6} \cdot T_{1}$ & $10964 \leq 1$ & 1997 \\
\hline$\left.r \Lambda_{6}\right) 90$ & $1 \cdot \Lambda_{6} r \cdot r$ & $170,4 Y 1$ & $199 V$ \\
\hline EMเVTr & IYYG $Y \leq r$ & IV. GVYY & 1991 \\
\hline ET,Yדו & I 1 & $1 V_{6} 9 V^{\prime}$ & 1999 \\
\hline$\varepsilon 9, \wedge \wedge \wedge$ & 1516 TYT & $1 \wedge \varepsilon 6 \leqslant 7$. & $r \ldots$ \\
\hline$\left.\sum \nearrow_{6} \leqslant\right) V$ & 1Y0,50Y & INVGYqY & $r \ldots 1$ \\
\hline $0.6 \vee 0$. & $1 Y V_{6} 19 \leq$ & r. r.VV9 & $r \ldots r$ \\
\hline $7 \Lambda_{6} Y 11$ & $1 \leq V 697 \pi$ & $Y 1161991$ & $r \cdot r$ \\
\hline 77610. & $10.691 \mathrm{r}$ & rTH,TYI & $r \ldots \varepsilon$ \\
\hline VYGVTO & ITVGY. & $r \leqslant r_{6} \vee 9$. & $r \ldots o$ \\
\hline$V r_{6} 0 V \lambda$ & $I V \wedge_{6} 0 \wedge V$ & YOT,TYI & $r \ldots T$ \\
\hline 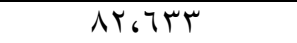 & 1196.94 & rצT.TOY & $r \ldots r$ \\
\hline $19.07 \%$ & 191.017 & Y79.VTV & $r \ldots \Lambda$ \\
\hline $9161 \mathrm{~V}$. & Y.T.VTE & YフT.TYO & $r .99$ \\
\hline $9 \cdot 64 \cdot 1$ & Y. Y.V10 & YA.6rOT & Y.1. \\
\hline $1 \cdots .911$ & YIV6) & $r \wedge \wedge, r \wedge \Lambda$ & $r .11$ \\
\hline $1 \cdot v_{6} \leq \leq 9$ & $Y 1 V_{6} \cdot 71$ & $r 90,0 \ldots$ & $r .1 Y$ \\
\hline $1 \cdot v_{6} 1 \leq 0$ & $r M T_{6} .1 T$ & $r \wedge 9,9 \vee q$ & $r .1 T$ \\
\hline $167 V / 6 \wedge 97$ & $56 r 9.60 \wedge r$ & $7 . \vee 9.6 \vee \wedge V$ & الإجمالى \\
\hline \% ro & $\% 70$ & $\% 1 \ldots$ & منوسط النسبة \\
\hline
\end{tabular}

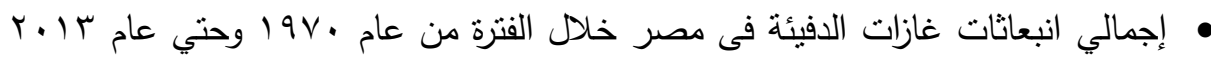
يبلغ حوالي ^، 7 مليون طن. • إجمالي انبعاثات غاز ثاني أكسيد الكربون في مصر خلال الفترة من عام • 19 وحتي عام rا • ب يبلغ وس، مليون طن. • نسبة انبعاثات غاز ثاني أكسيد الكربون إلي إجمالي انبعاثات غازات الدفيئة فى مصر

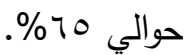


19V. • من محطات انتاج الكهرباء خلال الفترة من عام CO2

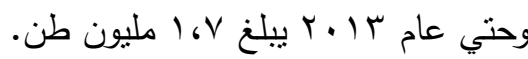
• متوسط نسبة انبعاثات غاز CO2 من محطات انتاج الكهرباء إلي إجمالي انبعاثات

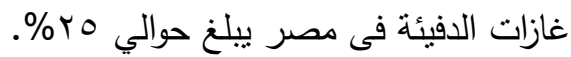

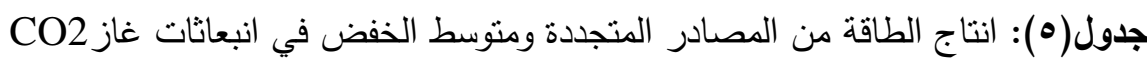

\begin{tabular}{|c|c|c|}
\hline 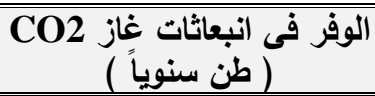 & ( جيجاوات ساعة سنة المنوياً) & نوع المشروع \\
\hline $1 r 04$ & $Y, V V$ & منتوسط الميجاوات (رياح ) \\
\hline 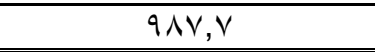 & $r, \cdot 1$ & متوسط الميجاوات ( شمسية ) \\
\hline
\end{tabular}

• منوسط انتاج الميجاوات من الطاقة المتجددة ( رياح ) يبلغ ب,VV جيجاوات ساعة سنوياً،

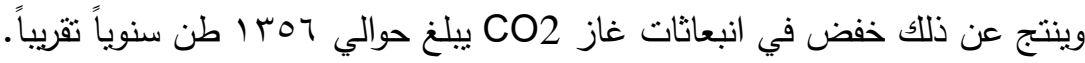

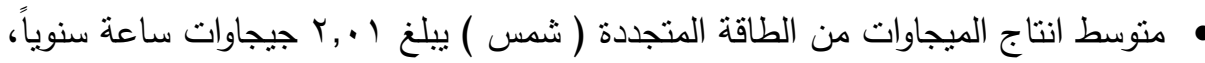

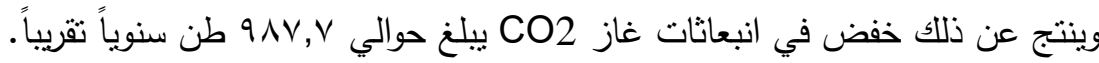

\section{المنائية}

ينبغي أن ينم النظر للطاقات المتجددة علي أنها بديل حقيقي لمصادر الطاقة الاحفورية،

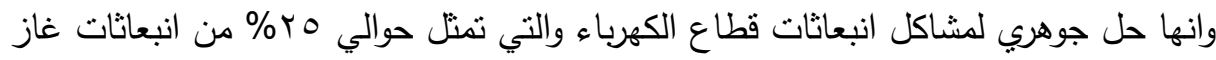
CO2 في مصر، ومن خلال تحليل تطور انتاج الطاقة الكهربائية ومصادر انتاجها ومعدل

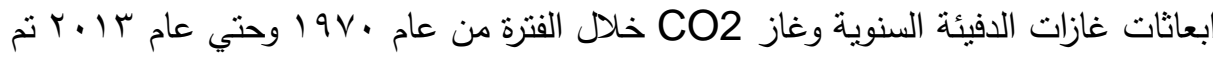

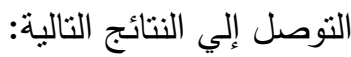

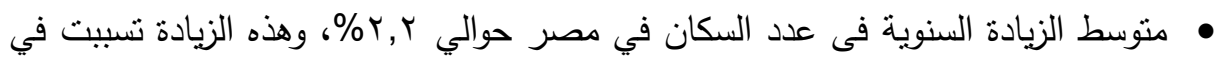

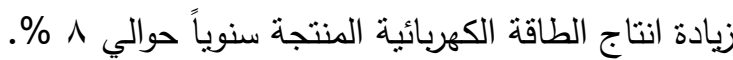

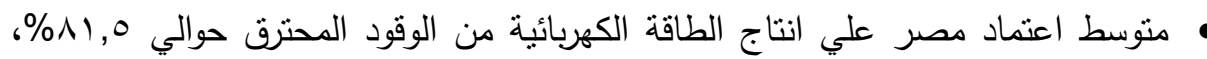
ولكن هذه النسبة ارتفعت لتصبح حوالي .9\% في أخر خمس سنوات ويزداد في الارتفاع بسبب التأخر في تتفيذ استراتيجية الطاقة المتجددة وأيضاً انخفاض انتاج المحطات المائية. 


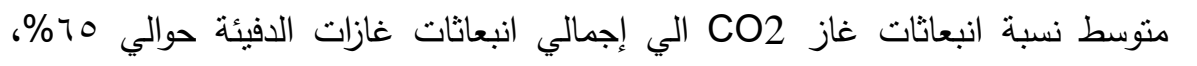
منوسط نسبة انبعاثات غاز CO2 من محطات انتاج الكهرباء الي إجمالي انبعاثات

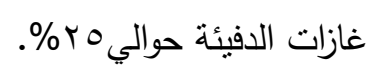

من المتوقع في حالة الاستمرار في زيادة عدد السكان في مصر، حيث من المتوقع زيادة

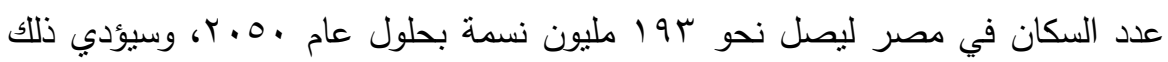

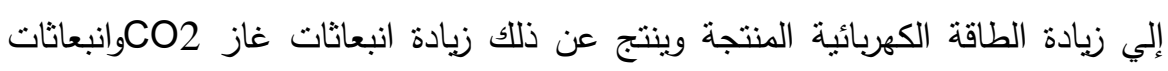
غازات الدفيئة في حالة استمرار مزيج انتاج الطاقة الكهربائية كما هو حالياً. من خلا استخدام برنامج RET Screen في قياس أثز استبدال مشروع طاقة أحفورية بمشروع طاقة متجددة ( شمس أو رياح )، حيث يتوقف اختبار نوع التكنولوجيا علي في فئرن

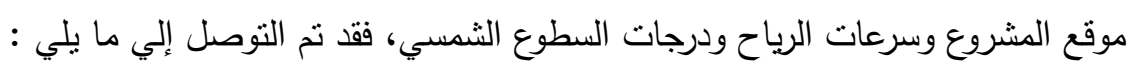

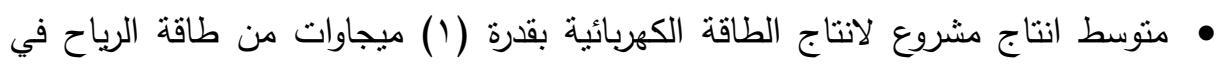

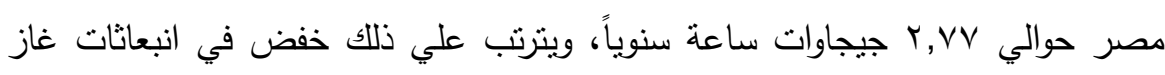
CO2 • متوسط انتاج مشروع لانتاج الطاقة الكهربائية بقدرة (1) ميجاوات من الطاقة الثمسية في

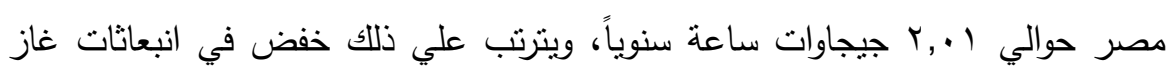
C^v, CO2

\section{التحوسياهي}

• وضع استراتيجية ملزمة لخفض انبعاثات غازات الدفيئة في مصر كثنيلها من دول العالم

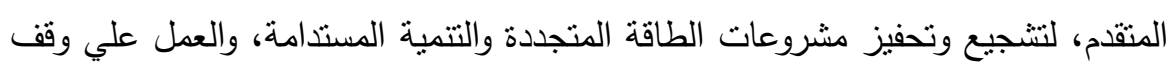
الانبعاثات الملوثة للبيئة والحد من التغيرات المناخية العالمية. • وضع خطط قصيرة الاجل لتنفيذ ومتابعة مشروعات الطاقة المتجدة، ونتيسيير اقبال المستثمرين علي تتفيذ نلك المشروعات. • وضع خطط ملزمة للطاقات المتجددة بشأن الاعفاءات الضريبية والجمركية لتشجيع

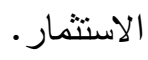


• فتح سوق بيع الطاقة الكهربائية من خلال تفعيل اليات السوق الحر للعرض والطلب .

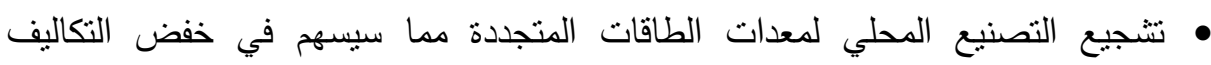
الاستثمارية اللازمة لإنثاء هذه المشروعات. تتفيذ خطط الربط بالدول العربية والتوسع في الربط مع دول العالم لنتجيع انتاج الطاقة من

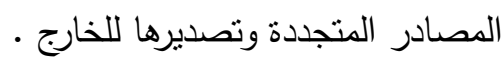
• تحسين عمل شبكات نقل الكهرباء لتخفيض الفقد في الطاقة المنتجة ورفع النسب الفنية لمساهمة الطاقات المتجددة في نسب الانتاج والربط بالثبكة.

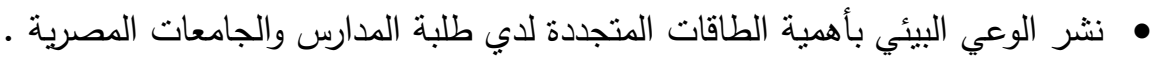

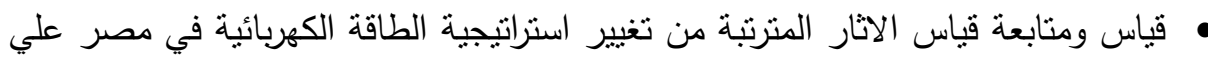

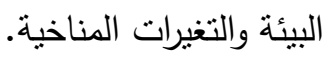
الاهتمام ببرامج ترشيد الطاقة ورفع كفاءة الطاقة في مصر لتقليل استهلاكى الطاقة الكهربائية. تشجيع مؤسسات التمويل المصرية علي تمويل مشروعات الطاقة المتجدة، من خلال تقديم حزم تمويلية ميسرة . الطاقة المتجددة ليست في إنثاء محطات انتاج الكهرباء فقط ولكن يمكن استخدامها في أغراض التسخين وتحلية المياة والعديد من مجالات الانتاج المختلفة.

\section{angll}

إبراهيم الغيطاني، وأمل عبد الغني(Y Y • Y): أفاق الطاقة المتجدة في مصر ، فرص الخروج

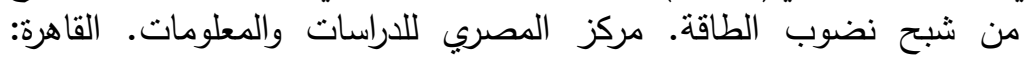

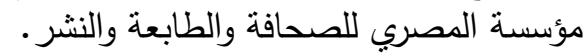

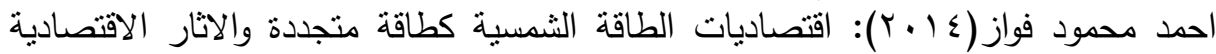
لاستثمارها في مصر • رسالة دكتوراة، جامعة عين شمس، كلية التجارة، القاهرة.

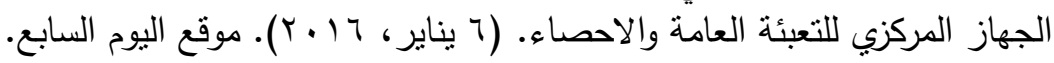

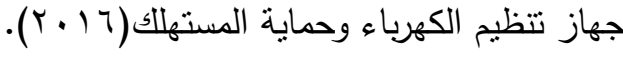


حدة فروحات(r ( †): الطاقات المتجددة كمدخل لتحقيق التتمية المستدامة في الجزائر.

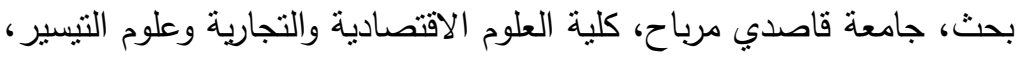

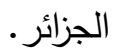

محرم عبد الكريم(7 ( • ب): الطاقة الثمسية. القاهرة، القاهرة، مصر : المكتبة الالكترونية.

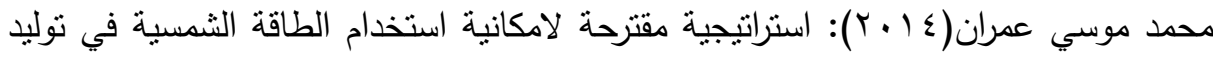

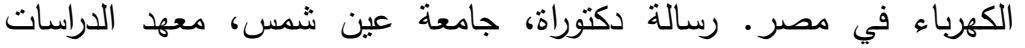

والبحوث البيئية، القاهرة.

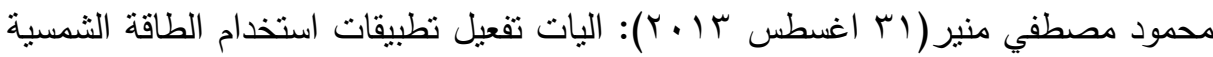

في ايجاد تتمية حضرية مستدامة. جامعة القاهرة، كلية التخطيط الاقليمي

$$
\text { والعمراني، القاهرة. }
$$

American petroleumInstitute. (2017): Climate Change and Energy. API Energy.

Climate action network. (2018): Climate change performance index. New climate institute. Bonn: German watch.

Commission, E. (2006): Strengths, weaknesses, opportunities and threats in energy. Energy Research. Retrieved 2006, from http://europa.eu.int/comm/research/rtdinfo/index_en.html

IAEA. (2016): Climate change and Nuclear power. Non-serial Publications, International Atomic Energy Agency, Vienna. Retrieved 2016, from Web: www.iaea.org/OurWork/ST/NE/Pess/

IEA. (2015): Energy and climate change. Special Report. Retrieved from www.worldenergyoutlook.org/energyclimate

IRENA. (2014): Estimating the Renewable Energy Potential in Africa. IRENA, A GIS-based approach. Germany: IRENA.

Katainen, J. (2013, march 20): National Energy and Climate Strategy. Government Report to Parliament, Finland. Retrieved from https://www.tem.fi/.../Energia-_ja_ilmastostrategia_nettij...

Meehl, Gerald. (2004, October 1): Combinations of Natural and AnthropogenicForcing in Twentieth Century Climate. Journal of Climate, 17, 7. Retrieved from http://acacia.ucar.edu/ccr/publications/meehl_additivity.pdf

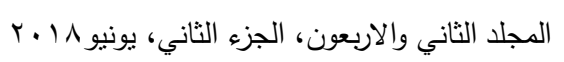


Pew Center. (2012): Climate change 101. Wilson: PEW center. Retrieved 2012, from https://www.pewcenteronthestates.prg

Taylor; (2016): Energy sources, part B: Economics, Planning, and Policy. Taylor \& Francis Group.

UNEP. (2016): Global Trends in Renewable energy investment 2016. Frankfurt: Frankfurt School-UNEP Collaborating Centre for Climate \& Sustainable Energy Finance.

\title{
USE RENEWABLE ENERGY SOURCES IN EGYPT TO REDUCE CLIMATE CHANGE
}

\author{
Fanous, N. A. ${ }^{(1)}$; El Kasas, H. I. $^{(2)}$; Omar, A. A. M. ${ }^{(3)}$ \\ and Ramadan, Hala, M. M.
}

1) Faculty of Commerce, Ain Shams University 2) Institute of Environmental Studies \& Research, Ain Shams University 3) New \& Renawable Energy Athourity.

\begin{abstract}
The main Goal of this study is benefiting from Egypt's clear richness in renewable sources of energy, especially sun and wind, in order to reduce climatic changes that affect many aspects of life and biological diversity.

The importance of the study is that it deals with a topic related to securing Egypt's energy supplies as well as the impact of sources of access to this energy on life. The study also stressed the importance of benefiting from the sources of renewable energy, which is a natural resource enjoyed by Egypt and should be exploited optimally for its The effects varied and varied on health, climate, environment and economy.
\end{abstract}

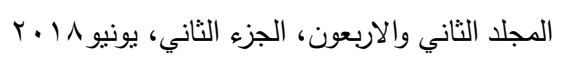


The study showed that fossil fuels still dominate the energy sources in Egypt and the world, and that it is one of the main causes of global warming.

The study showed the rates of development of the population increase and the increase in the production of electricity, the sources of production of this energy, and the development of emissions of greenhouse gases and $\mathrm{CO} 2$ gas in the world and Egypt, through historical data from 1970 to 2013.

The study also made a comparison between the global ratios reached and the percentages within the Arab Republic of Egypt for the same period of time.

The study also showed the global change in temperature from 1880 to 2013 , to show the global change in global temperatures and to stir up the different aspects of life.

In the end, the study presented a proposal for renewable energy management through a mathematical model that measures the impact of switching fossil energy sources to renewable energy sources on climate change. The model was built on two axes:

- First: the results of analysis and study of historical data. From World Bank - Data Bank.

- Second: Using RET Screen program to measure the results of replacing conventional energy projects with renewable energies.

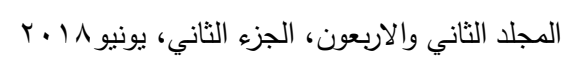

